\title{
AN EXTENSION OF TWO BASIC RESULTS IN REAL ANALYSIS
}

\author{
DORIN ERVIN DUTKAY, CONSTANTIN P. NICULESCU, AND FLORIN POPOVICI
}

\begin{abstract}
Based on the existence of well behaved partitions, we extend the Denjoy-Bourbaki Theorem and Leibniz-Newton Formula to a context where the lack of derivability is supplied by the property of negligible semivariation.
\end{abstract}

\section{INTRODUCTION}

In what follows $[a, b]$ denotes a nondegenerate compact interval and $E$ denotes a Banach space.

A subpartition of $[a, b]$ is a collection $\mathcal{P}=\left(I_{k}\right)_{k=1}^{n}$ of nonoverlapping closed intervals in $[a, b]$; if $\cup_{k} I_{k}=[a, b]$, we say that $\mathcal{P}$ is a partition. A tagged subpartition of $[a, b]$ is a collection of ordered pairs $\left(I_{k}, t_{k}\right)_{k=1}^{n}$ consisting of intervals $I_{k}$, that form a subpartition of $[a, b]$, and tags $t_{k} \in I_{k}$, for $k=1, \ldots, n$. If $\delta$ is a gauge (that is, a positive function) on a subset $A \subset[a, b]$ we say that a tagged subpartition $\left(I_{k}, t_{k}\right)_{k=1}^{n}$ is $(\delta, A)$-fine if all tags $t_{k}$ belong to $A$ and $I_{k} \subset\left(t_{k}-\delta\left(t_{k}\right), t_{k}+\delta\left(t_{k}\right)\right)$ for $k=1, \ldots, n$. A result known as Cousin's Lemma asserts the existence of $(\delta,[a, b])$ fine tagged partitions for each $\delta:[a, b] \rightarrow(0, \infty)$. See [1], page 11. This result is equivalent to many other basic results such as the Fundamental Lemma of Analysis on $\mathbb{R}$ (see [8]). In what follows we shall need a slightly more general version of Cousin's Lemma:

Lemma 1. Let $\delta$ be a gauge on $[a, b]$ and assume that $\mathcal{A}$ is a family of subintervals $\left[x^{\prime}, x^{\prime \prime}\right] \subset[a, b]$ which satisfies the following two conditions:

i) for every $z \in[a, b)$ and every $x^{\prime} \in(z-\delta(z), z] \cap[a, b]$ there exists $x^{\prime \prime} \in(z, b]$ such that $\left[x^{\prime}, x^{\prime \prime}\right] \in \mathcal{A}$;

ii) for every $x^{\prime} \in(b-\delta(b), b) \cap[a, b]$, the interval $\left[x^{\prime}, b\right]$ belongs to $\mathcal{A}$.

Then there exists a partition of $[a, b]$ consisting of intervals in $\mathcal{A}$.

Proof. Consider the set $\mathcal{C}$ of all points $c$ of $[a, b]$ such that $[a, c]$ admits a partition consisting of intervals in $\mathcal{A}$. Put $z=\sup \mathcal{C}$. According to $i$ ), $z>a$. By reductio ad absurdum we infer that actually $z=b$. Then $i i$ ) assures that $b \in \mathcal{C}$.

The original result of Cousin corresponds to the case where $\mathcal{A}$ is the family of all nondegenerate intervals $\left[x^{\prime}, x^{\prime \prime}\right]$ such that

$$
\left[x^{\prime}, x^{\prime \prime}\right] \subset(z-\delta(z), z+\delta(z)) \cap[a, b] \text { for some } z \in[a, b] .
$$

2000 Mathematics Subject Classification. Primary 26A24; 26A39; Secondary 26D10; 26A46.

Key words and phrases. Dini derivative, negligible variation, Mean Value Theorem, LeibnizNewton Formula.

Published in vol.: Mathematical Analysis and Applications, AIP Conference Proceedings Volume 835, pp. 48-57, American Institute of Physics, Melville, New York, 2006 (V. D. Rădulescu and C. P. Niculescu, Editors). ISBN 0-7354-0328-7. 
A related result, also extending Cousin's Lemma, is as follows:

Lemma 2. Let $\delta$ be a gauge on $[a, b]$ and assume that $\mathcal{A}$ is a family of subintervals $\left[x^{\prime}, x^{\prime \prime}\right] \subset[a, b]$ which satisfies the following three conditions:

i) there is $x^{\prime \prime} \in(a, b]$ such that $\left[a, x^{\prime \prime}\right] \in \mathcal{A}$;

ii) for every $z \in(a, b]$ and every $x^{\prime} \in(z-\delta(z), z) \cap[a, b]$, there is $x^{\prime \prime} \in[z, b]$ such that $\left[x^{\prime}, x^{\prime \prime}\right] \in \mathcal{A}$.

iii) for every $\left[x^{\prime}, x^{\prime \prime}\right] \in \mathcal{A}$ with $x^{\prime \prime}<b$ there is $y \in\left(x^{\prime \prime}, b\right]$ such that $\left[x^{\prime}, y\right] \in \mathcal{A}$.

Then there exists a partition of $[a, b]$ consisting of intervals in $\mathcal{A}$.

The two lemmata above are instrumental in our extension of the following two results in Real Analysis:

(DB) The Denjoy-Bourbaki Theorem (which in turn is a generalization of the Mean Value Theorem). This theorem was first published in [2], p. 2324, with an argument adapted from a celebrated paper of A. Denjoy [4], dedicated to the Dini derivatives. A nice account on it is available in [5], Ch. 8, Section 5.2.

(LN) The Leibniz-Newton Formula for Lebesgue integrable right derivatives. See [7], p. 298-299, or [12].

A function $F:[a, b] \rightarrow E$ is said to have negligible variation on a set $A \subset[a, b]$ (and we write $\left.F \in N V_{A}([a, b], E)\right)$ if, for every $\varepsilon>0$ there exists a gauge $\delta_{\varepsilon}$ on $A$ such that if $\mathcal{D}=\left\{\left(\left[u_{k}, v_{k}\right]\right), t_{k}\right\}_{k=1}^{n}$ is any $\left(\delta_{\varepsilon}, A\right)$-fine tagged subpartition of $[a, b]$, then

$$
\operatorname{Var}(F ; \mathcal{D})=\sum_{k=1}^{n}\left\|F\left(v_{k}\right)-F\left(u_{k}\right)\right\|<\varepsilon .
$$

Analogously, $F:[a, b] \rightarrow E$ is said to have negligible semivariation on a set $A \subset[a, b]$ (and we write $F \in N S V_{A}([a, b], E)$ ) if, for every $\varepsilon>0$ there exists a gauge $\delta_{\varepsilon}$ on $A$ such that if $\mathcal{D}=\left\{\left(\left[u_{k}, v_{k}\right]\right), t_{k}\right\}_{k=1}^{n}$ is any $\left(\delta_{\varepsilon}, A\right)$-fine tagged subpartition of $[a, b]$, then

$$
\left\|\sum_{k=1}^{n}\left(F\left(v_{k}\right)-F\left(u_{k}\right)\right)\right\|<\varepsilon .
$$

For real-valued functions the two notions agree,

$$
N S V_{A}([a, b], \mathbb{R})=N V_{A}([a, b], \mathbb{R}) .
$$

Clearly, if $F \in N V_{A}([a, b], E)$, then $F$ is continuous at every point of $A$. Conversely, if $C$ is a countable set in $[a, b]$ and $F:[a, b] \rightarrow E$ is continuous at every point of $C$, then $F \in N V_{C}([a, b], E)$. However, when $Z \subset[a, b]$ is a Lebesgue negligible set, there are continuous functions on $[a, b]$ that do not belong to $N S V_{Z}([a, b], E)$. See [1], page 233, for an example.

Given a scalar function $\varphi:[a, b] \rightarrow \mathbb{R}$, one can attach to it the Dini derivatives. In what follows we are interested in the upper right derivative,

$$
D^{+} \varphi(x)=\limsup _{h \downarrow 0} \frac{\varphi(x+h)-\varphi(x)}{h} \quad \text { for } x \in[a, b)
$$

and the lower left derivative,

$$
D_{-} \varphi(x)=\liminf _{h \uparrow 0} \frac{\varphi(x+h)-\varphi(x)}{h} \text { for } x \in(a, b] .
$$


We are now in a position to state our generalization of the Denjoy-Bourbaki Theorem:

Theorem 1. Let $F:[a, b] \rightarrow E$ and $\varphi:[a, b] \rightarrow \mathbb{R}$ be two continuous functions which fulfil the following three conditions with respect to a suitable disjoint decomposition $[a, b]=A_{1} \cup A_{2} \cup A_{3}$ :

i) $F$ and $\varphi$ have negligible semivariation on $A_{1}$;

ii) $F$ has a right derivative $F_{+}^{\prime}$ at all points of $A_{2}$ and $\left\|F_{+}^{\prime}\right\| \leq D^{+} \varphi$ on $A_{2}$;

iii) $F$ has a left derivative $F_{-}^{\prime}$ at all points of $A_{3}$ and $\left\|F_{-}^{\prime}\right\| \leq D_{-} \varphi$ on $A_{3}$.

Then

$$
\|F(b)-F(a)\| \leq \varphi(b)-\varphi(a) .
$$

The details will be given in Section 2 .

The classical case corresponds to the situation where $A_{1}$ is at most countable and both $F$ and $\varphi$ have a right derivative at all points of $A_{2}=[a, b) \backslash A_{1}$. In that case the condition $i$ ) is automatically satisfied.

Under the assumption that $F$ and $\varphi$ are both differentiable outside $A_{1}$, Theorem 1 has been proved in [9].

The Dini derivatives take values in $\overline{\mathbb{R}}$. Theorem 1 proves that a continuous function $\varphi:[a, b] \rightarrow \mathbb{R}$ cannot have an infinite upper right derivative at all points, even excepting a countable subset (or, more generally, a subset on which $\varphi$ has negligible variation).

The case where $F=0$ in Theorem 1 is an improvement of an old criterion of monotonicity mentioned by S. Saks in his monograph [11], p. 204:

Corollary 1. Let $\varphi:[a, b] \rightarrow \mathbb{R}$ be a continuous functions for which there exists a disjoint decomposition $[a, b]=A_{1} \cup A_{2} \cup A_{3}$ such that:

i) $\varphi$ has negligible variation on $A_{1}$;

ii) $D^{+} \varphi \geq 0$ on $A_{2}$;

iii) $D_{-} \varphi \geq 0$ on $A_{3}$.

Then $\varphi$ is nondecreasing.

An immediate consequence of Theorem 1 (for $\varphi(x)=M(x-a)$ ) is the following:

Corollary 2. Let $F:[a, b] \rightarrow E$ be a continuous function for which there exists a subset $A \subset[a, b]$ such that:

i) $F$ has negligible semivariation on $A$;

ii) $F$ has a right derivative $F_{+}^{\prime}$ at all points of $[a, b) \backslash A$ and $\left\|F_{+}^{\prime}\right\| \leq M$ on $[a, b) \backslash A$.

Then

$$
\|F(b)-F(a)\| \leq M(b-a) .
$$

Corollary 1 allows us to retrieve the following classical result due to L. Scheefer:

Proposition 1. Suppose that $F:[a, b] \rightarrow \mathbb{R}$ and $G:[a, b] \rightarrow \mathbb{R}$ are two continuous functions which admit finite upper right derivatives except on a countable subset $C$ and $D^{+} F=D^{+} G$ at all points of $[a, b] \backslash C$. Then $F-G$ is a constant function.

Proof. In fact, from $G=(G-F)+F$ we infer that

$$
D^{+} G \leq D^{+}(G-F)+D^{+} F,
$$

so by our hypothesis we get $D^{+}(G-F) \geq 0$ on $[a, b] \backslash C$. As $C$ is countable, $G-F$ has negligible semivariation on $C$ and thus $G-F$ is nondecreasing by Corollary 1 . Changing the role of $F$ and $G$ we conclude that $F-G$ is constant. 
The discussion above suggests us to consider the following generalization of the concept of a primitive function:

Definition 1. Given a function $f:[a, b] \rightarrow E$, by a right primitive of $f$ we mean any continuous function $F:[a, b] \rightarrow E$ which verifies the following two conditions:

i) $F$ has a right derivative $F_{+}^{\prime}$ at all points of $[a, b]$ except for a Lebesgue negligible subset $A$ on which $F$ has negligible semivariation;

ii) $F_{+}^{\prime}=f$ on $[a, b] \backslash A$.

The concept of a left primitive can be introduced in the same manner.

By using Lemma 1 one can prove that any two right primitives of a function differ by a constant.

The importance of Definition 1 above is outlined by the following generalization of the classical Leibniz-Newton Formula:

Theorem 2. Let $f:[a, b] \rightarrow E$ be a function which is integrable in the sense of Henstock and Kurzweil and admits right primitives. Then

$$
\int_{a}^{b} f(t) d t=F(b)-F(a)
$$

for every right primitive $F$ of $f$.

Recall that a function $f:[a, b] \rightarrow E$ is said to be integrable in the sense of Henstock and Kurzweil if there exists a vector $I \in E$ such that for every $\varepsilon>0$ one can find a gauge $\delta:[a, b] \rightarrow(0, \infty)$ such that for every $(\delta,[a, b])$-fine tagged partition $\left\{\left(\left[u_{k}, v_{k}\right]\right), t_{k}\right\}_{k=1}^{n}$ of $[a, b]$, we have

$$
\left\|I-\sum_{k=1}^{n} f\left(t_{k}\right)\left(v_{k}-u_{k}\right)\right\|<\varepsilon .
$$

The vector $I$ is unique with the above properties. It represents the integral of $f$ over $[a, b]$, usually denoted by $\int_{a}^{b} f(t) d t$.

In the context of Lebesgue integrability, a special case of Theorem 2 has been proved by E. Hewitt and K. Stromberg [7]. See also [12] for a simple proof. A nice application is the fact that

$$
\int_{a}^{b} f_{+}^{\prime}(t) d t=f(b)-f(a)
$$

for every continuous convex function $f:[a, b] \rightarrow \mathbb{R}$.

Theorem 2 yields Corollary 2. This is clear in the case where $E=\mathbb{R}$. In the general case, notice that we may restrict to the case of real Banach spaces and then use the formula

$$
(h \circ F)_{+}^{\prime}=h \circ F_{+}^{\prime} \quad \text { for every } h \in E^{\prime} .
$$

In Section 3 we shall prove a result which extends Theorem 2.

Finally, it is worth noticing that the entire theory above can be extended to the framework of relative derivatives. Given a function $F:[a, b] \rightarrow E$, a subset $A \subset[a, b]$ and a point $z \in[a, b]$ (assumed to be a limit point of $A$ ), we define the derivative of $F$ at $z$ relative to $A$ by the formula

$$
F^{\prime}(z ; A)=\lim _{\substack{x \rightarrow z \\ x \in A}} \frac{F(x)-F(z)}{x-z}
$$


provided that the limit exists. In a similar manner one can define the relative Dini derivatives $D^{+} F(z ; A), D_{+} F(z ; A), D^{-} F(z ; A)$ and $D_{-} F(z ; A)$. The details concerning the extension of Theorems 1 and 2 to this framework will be presented elsewhere.

\section{Proof of Theorem 1}

Suppose there is given $\varepsilon>0$.

By the assumption $i)$, there exists a gauge $\delta: A_{1} \rightarrow(0, \infty)$ such that for every $\left(\delta, A_{1}\right)$-fine subpartition $\left(\left[u_{k}, v_{k}\right]\right)_{k=1}^{n}$ we have

$$
\left\|\sum_{k=1}^{n}\left(F\left(v_{k}\right)-F\left(u_{k}\right)\right)\right\| \leq \varepsilon / 4 \text { and } \sum_{k=1}^{n}\left|\varphi\left(v_{k}\right)-\varphi\left(u_{k}\right)\right|<\varepsilon / 4 .
$$

We shall denote by $\mathcal{A}_{1}$ the family of all subintervals $\left[x^{\prime}, x^{\prime \prime}\right]$ of $[a, b]$ such that

$$
\left[x^{\prime}, x^{\prime \prime}\right] \subset(y-\delta(y), y+\delta(y))
$$

for suitable $y \in\left[x^{\prime}, x^{\prime \prime}\right] \cap A_{1}$.

According to $i i)$, for each $z \in A_{2}$,

$$
\liminf _{x \rightarrow z+}\left(\left\|\frac{F(x)-F(z)}{x-z}\right\|-\frac{\varphi(x)-\varphi(z)}{x-z}\right)=\left\|F_{+}^{\prime}(z)\right\|-D^{+} \varphi(z) \leq 0,
$$

which yields an $y \in(z, b]$ such that

$$
\left\|\frac{F(y)-F(z)}{y-z}\right\|-\frac{\varphi(y)-\varphi(z)}{y-z}<\frac{\varepsilon}{2(b-a)},
$$

equivalently,

$$
\alpha=\frac{\varepsilon}{2(b-a)}(y-z)-\|F(y)-F(z)\|+(\varphi(y)-\varphi(z))>0 .
$$

Since the functions $F$ and $\varphi$ are continuous at $z$, there exists a positive number $\delta_{1}(z)$ such that for every $x^{\prime} \in\left(z-\delta_{1}(z), z\right] \cap[a, b]$ we have

$$
\left\|F\left(x^{\prime}\right)-F(z)\right\|<\alpha / 4 \text { and }\left|\varphi\left(x^{\prime}\right)-\varphi(z)\right|<\alpha / 4
$$

and for every $x^{\prime \prime} \in\left[y, y+\delta_{1}(z)\right) \cap[a, b]$ we have

$$
\left\|F\left(x^{\prime \prime}\right)-F(y)\right\|<\alpha / 4 \text { and }\left|\varphi\left(x^{\prime \prime}\right)-\varphi(y)\right|<\alpha / 4 .
$$

Then

$$
\frac{\varepsilon}{2(b-a)}\left(x^{\prime \prime}-x^{\prime}\right)-\left\|F\left(x^{\prime \prime}\right)-F\left(x^{\prime}\right)\right\|+\left(\varphi\left(x^{\prime \prime}\right)-\varphi\left(x^{\prime}\right)\right)>\alpha-4 \cdot \alpha / 4=0,
$$

that is,

$$
\left\|F\left(x^{\prime \prime}\right)-F\left(x^{\prime}\right)\right\|-\left(\varphi\left(x^{\prime \prime}\right)-\varphi\left(x^{\prime}\right)\right)<\frac{\varepsilon}{2(b-a)}\left(x^{\prime \prime}-x^{\prime}\right) .
$$

We denote by $\mathcal{A}_{2}$ be the family of all intervals $\left[x^{\prime}, x^{\prime \prime}\right]$ which appear this way.

Similarly, for every $z \in A_{3}$,

$$
\limsup _{x \rightarrow z-}\left(\left\|\frac{F(x)-F(z)}{x-z}\right\|-\frac{\varphi(x)-\varphi(z)}{x-z}\right)=\left\|F_{-}^{\prime}(z)\right\|-D_{-} \varphi(z) \leq 0,
$$

and thus there exists a positive number $\delta_{1}(z)$ such that for every $x^{\prime} \in\left(z-\delta_{1}(z), z\right) \cap$ $[a, b]$, we have

$$
\left\|F(z)-F\left(x^{\prime}\right)\right\|-\left(\varphi(z)-\varphi\left(x^{\prime}\right)\right)<\frac{\varepsilon}{2(b-a)}\left(z-x^{\prime}\right) .
$$


Then

$$
\beta=\frac{\varepsilon}{2(b-a)}\left(z-x^{\prime}\right)-\left\|F(z)-F\left(x^{\prime}\right)\right\|+\left(\varphi(z)-\varphi\left(x^{\prime}\right)\right)>0 .
$$

Since $F$ and $\varphi$ are continuous on $[a, b]$, we can find a positive number $\delta_{2}$ such that

$$
\left\|F\left(x^{\prime \prime}\right)-F(z)\right\|<\beta / 2 \text { and }\left|\varphi\left(x^{\prime \prime}\right)-\varphi(z)\right|<\beta / 2
$$

for every $x^{\prime \prime} \in\left[z, z+\delta_{2}\right) \cap[a, b]$. Then

$$
\frac{\varepsilon}{2(b-a)}\left(x^{\prime \prime}-x^{\prime}\right)-\left\|F\left(x^{\prime \prime}\right)-F\left(x^{\prime}\right)\right\|+\left(\varphi\left(x^{\prime \prime}\right)-\varphi\left(x^{\prime}\right)\right)>\beta-2 \cdot \beta / 2=0
$$

that is,

$$
\left\|F\left(x^{\prime \prime}\right)-F\left(x^{\prime}\right)\right\|-\left(\varphi\left(x^{\prime \prime}\right)-\varphi\left(x^{\prime}\right)\right)<\frac{\varepsilon}{2(b-a)}\left(x^{\prime \prime}-x^{\prime}\right) .
$$

This reasoning yields a new family $\mathcal{A}_{3}$ of subintervals of $[a, b]$.

The family

$$
\mathcal{A}=\mathcal{A}_{1} \cup \mathcal{A}_{2} \cup \mathcal{A}_{3}
$$

verifies the hypothesis of Lemma 2 and thus there exists a partition $\mathcal{D}=\left(\left[x_{i}, x_{i+1}\right]\right)_{i=0}^{n-1}$ of $[a, b]$ into subintervals of $\mathcal{A}$.

By (2.1), (2.2) and (2.3), we get

$$
\begin{aligned}
& \|F(b)-F(a)\|-(\varphi(b)-\varphi(a)) \leq \\
& \leq\left\|\sum_{\left[x_{i}, x_{i+1}\right] \in \mathcal{A}_{1}}\left(F\left(x_{i+1}\right)-F\left(x_{i}\right)\right)\right\|+\sum_{\left[x_{i}, x_{i+1}\right] \in \mathcal{A}_{1}}\left|\varphi\left(x_{i+1}\right)-\varphi\left(x_{i}\right)\right| \\
& +\sum_{\left[x_{i}, x_{i+1}\right] \in \mathcal{A} \backslash \mathcal{A}_{1}}\left(\left\|F\left(x_{i+1}\right)-F\left(x_{i}\right)\right\|-\left(\varphi\left(x_{i+1}\right)-\varphi\left(x_{i}\right)\right)\right) \\
& <\frac{\varepsilon}{4}+\frac{\varepsilon}{4}+\frac{\varepsilon}{2(b-a)} \sum_{\left[x_{i}, x_{i+1}\right] \in \mathcal{A} \backslash \mathcal{A}_{1}}\left(x_{i+1}-x_{i}\right) \leq \varepsilon,
\end{aligned}
$$

which means that $\|F(b)-F(a)\|-(\varphi(b)-\varphi(a))<\varepsilon$. As $\varepsilon>0$ was fixed arbitrary, we conclude that $\|F(b)-F(a)\|-(\varphi(b)-\varphi(a)) \leq 0$.

\section{A General Leibniz-Newton Formula}

The aim of this section is to prove the following generalization of Theorem 2 :

Theorem 3. Let $F:[a, b] \rightarrow E$ and $f:[a, b] \rightarrow \mathbb{R}$ be two functions for which there exists a disjoint decomposition $[a, b]=A_{1} \cup A_{2} \cup A_{3}$ such that:

i) $F$ is continuous on $[a, b]$ and has negligible semivariation on $A_{1}$;

ii) $F$ has a right derivative $F_{+}^{\prime}$ at all points of $A_{2}$ and a left derivative $F_{-}^{\prime}$ at all points of $A_{3}$;

iii) $f$ is integrable in the sense of Henstock-Kurzweil and

$$
f(x)= \begin{cases}0 & \text { if } x \in A_{1} \\ F_{+}^{\prime}(x) & \text { if } x \in A_{2} \\ F_{-}^{\prime}(x) & \text { if } x \in A_{3}\end{cases}
$$

Then

$$
\int_{a}^{b} f(x) d x=F(b)-F(a) .
$$

When $A_{1}$ is Lebesgue negligible, the condition $f=0$ on $A_{1}$ can be removed. 
Proof. Let $\varepsilon>0$ be arbitrarily fixed. Since the function $f$ is integrable, there is a gauge $\delta_{1}:[a, b] \rightarrow(0, \infty)$ such that for every $\delta_{1}$-fine tagged partition $\mathcal{D}=$ $\left\{\left(\left[x_{i-1}, x_{i}\right]\right), t_{i}\right\}_{i=1}^{m}$ of $[a, b]$, we have

$$
\left\|\int_{a}^{b} f(x) d x-\sum_{i=1}^{m} f\left(t_{i}\right)\left(x_{i}-x_{i-1}\right)\right\|<\frac{\varepsilon}{2} .
$$

Since $F \in N S V_{A_{1}}([a, b], E)$, we can choose a gauge $\delta_{2}:[a, b] \rightarrow(0, \infty)$ such that $\delta_{2} \leq \delta_{1}$ on $A_{1}$ and for any $\left(\delta_{2}, A_{1}\right)$-fine tagged subpartition $\mathcal{D}=\left\{\left(\left[x_{i}^{\prime}, x_{i}^{\prime \prime}\right]\right), s_{i}\right\}_{i=1}^{n}$ of $[a, b]$, we have

$$
\left\|\sum_{i=1}^{n}\left(F\left(x_{i}^{\prime \prime}\right)-F\left(x_{i}^{\prime}\right)\right)\right\|<\frac{\varepsilon}{4} .
$$

We shall denote by $\mathcal{A}_{1}$ the family of all subintervals of $[a, b]$ for which there are points $z \in\left[x^{\prime}, x^{\prime \prime}\right] \cap A_{1}$ such that

$$
\left[x^{\prime}, x^{\prime \prime}\right] \subset\left(z-\delta_{2}(z), z+\delta_{2}(z)\right) .
$$

Clearly, $\mathcal{A}_{1}$ consists of $\delta_{1}$-fine intervals.

Suppose that $z \in A_{2}$. By $\left.i i\right)$, we can choose a number $\delta_{2}(z) \in\left(0, \delta_{1}(z)\right]$ such that

$$
y \in\left(z, z+\delta_{2}(z)\right) \cap[a, b] \text { implies }\left\|\frac{F(y)-F(z)}{y-z}-f(z)\right\|<\frac{\varepsilon}{4(b-a)} .
$$

The last inequality says that

$$
\alpha=\frac{\varepsilon}{4(b-a)}(y-z)-\|F(y)-F(z)-f(z)(y-z)\|>0,
$$

so that by the continuity of $F$ we may choose a number

$$
\delta_{3}(z, y) \in\left(0, \min \left\{z+\delta_{1}(z)-y, \frac{\alpha}{4(1+\|f(z)\|)}\right\}\right)
$$

for which

$$
x^{\prime} \in\left(z-\delta_{3}(z, y), z\right] \cap[a, b] \text { implies }\left\|F\left(x^{\prime}\right)-F(z)\right\|<\frac{\alpha}{4}
$$

and

$$
x^{\prime \prime} \in\left[y, y-\delta_{3}(z, y)\right) \cap[a, b] \text { implies }\left\|F\left(x^{\prime \prime}\right)-F(y)\right\|<\frac{\alpha}{4} .
$$

Therefore for all $x^{\prime} \in\left(z-\delta_{3}(z, y), z\right] \cap[a, b]$ and all $x^{\prime \prime} \in\left[y, y+\delta_{3}(z, y)\right) \cap[a, b]$ we have

$$
\frac{\varepsilon}{4(b-a)}\left(x^{\prime \prime}-x^{\prime}\right)-\left\|F\left(x^{\prime \prime}\right)-F\left(x^{\prime}\right)-f(z)\left(x^{\prime \prime}-x^{\prime}\right)\right\|>\alpha-4 \cdot \frac{\alpha}{4}=0
$$

and thus

$$
\left\|F\left(x^{\prime \prime}\right)-F\left(x^{\prime}\right)-f(z)\left(x^{\prime \prime}-x^{\prime}\right)\right\|<\frac{\varepsilon}{4(b-a)}\left(x^{\prime \prime}-x^{\prime}\right) .
$$

We shall denote by $\mathcal{A}_{2}$ the set of all intervals $\left[x^{\prime}, x^{\prime \prime}\right]$ that appear by the preceding reasoning.

Suppose that $z \in A_{3}$. By $\left.i i i\right)$, we can choose a number $\delta_{2}(z) \in\left(0, \delta_{1}(z)\right]$ such that

$$
y \in\left(z-\delta_{2}(z), z\right) \cap[a, b] \text { implies }\left\|\frac{F(y)-F(z)}{y-z}-f(z)\right\|<\frac{\varepsilon}{4(b-a)} .
$$


The last inequality says that

$$
\beta=\frac{\varepsilon}{4(b-a)}(z-y)-\|F(z)-F(y)-f(z)(z-y)\|>0,
$$

so that by the continuity of $F$ we may choose for each $x^{\prime} \in\left(z-\delta_{2}(z), z\right) \cap[a, b]$ a number

for which

$$
\delta_{3}\left(z, x^{\prime}\right) \in\left(0, \min \left\{\delta_{2}(z), \frac{\beta}{2(1+\|f(z)\|)}\right\}\right)
$$

$$
x^{\prime \prime} \in\left[z, z+\delta_{3}\left(z, x^{\prime}\right)\right] \cap[a, b] \text { implies }\left\|F\left(x^{\prime \prime}\right)-F(z)\right\|<\frac{\beta}{2} .
$$

Therefore for all $x^{\prime \prime} \in\left[z, z+\delta_{3}\left(z, x^{\prime}\right)\right] \cap[a, b]$ we have

$$
\frac{\varepsilon}{4(b-a)}\left(x^{\prime \prime}-x^{\prime}\right)-\left\|F\left(x^{\prime \prime}\right)-F\left(x^{\prime}\right)-f(z)\left(x^{\prime \prime}-x^{\prime}\right)\right\|>\beta-2 \cdot \frac{\beta}{2}=0
$$

and thus

$$
\left\|F\left(x^{\prime \prime}\right)-F\left(x^{\prime}\right)-f(z)\left(x^{\prime \prime}-x^{\prime}\right)\right\|<\frac{\varepsilon}{4(b-a)}\left(x^{\prime \prime}-x^{\prime}\right) .
$$

We shall denote by $\mathcal{A}_{3}$ the new set of intervals $\left[x^{\prime}, x^{\prime \prime}\right]$ that appear by the last reasoning.

The family of intervals

$$
\mathcal{A}=\mathcal{A}_{1} \cup \mathcal{A}_{2} \cup \mathcal{A}_{3}
$$

fulfils the hypotheses of Lemma 2 and thus there is a partition $\mathcal{D}=\left(\left[x_{i}, x_{i+1}\right]\right)_{i=0}^{n-1}$ of $[a, b]$ consisting of intervals of $\mathcal{A}$. Clearly, $\mathcal{D}$ is $\delta_{1}$-fine. By the relation (4) we get

$$
\begin{gathered}
\left\|F(b)-F(a)-\int_{a}^{b} f(x) d x\right\| \\
\leq\left\|\sum_{i=0}^{n-1}\left[F\left(x_{i+1}\right)-F\left(x_{i}\right)-f\left(z_{i}\right)\left(x_{i+1}-x_{i}\right)\right]\right\|+\left\|\sum_{i=0}^{n-1} f\left(z_{i}\right)\left(x_{i+1}-x_{i}\right)-\int_{a}^{b} f(x) d x\right\| \\
<\left\|\sum_{i=0}^{n-1}\left[F\left(x_{i+1}\right)-F\left(x_{i}\right)-f\left(z_{i}\right)\left(x_{i+1}-x_{i}\right)\right]\right\|+\frac{\varepsilon}{2} .
\end{gathered}
$$

On the other hand, by (5)-(7) and the fact that $\left.f\right|_{A_{1}}=0$, we get

$$
\begin{aligned}
& \left\|\sum_{i=0}^{n-1}\left[F\left(x_{i+1}\right)-F\left(x_{i}\right)-f\left(z_{i}\right)\left(x_{i+1}-x_{i}\right)\right]\right\| \\
& \leq\left\|\sum_{\left\{i \mid\left[x_{i}, x_{i+1}\right] \in \mathcal{A}_{1}\right\}}\left(F\left(x_{i+1}\right)-F\left(x_{i}\right)\right)\right\| \\
& +\sum_{\left\{i \mid\left[x_{i}, x_{i+1}\right] \in \mathcal{A} \backslash \mathcal{A}_{1}\right\}}^{\left\|F\left(x_{i+1}\right)-F\left(x_{i}\right)-f\left(z_{i}\right)\left(x_{i+1}-x_{i}\right)\right\|} \\
& \quad<\frac{\varepsilon}{4}+\frac{\varepsilon}{4(b-a)} \sum_{i=1}^{n-1}\left(x_{i+1}-x_{i}\right)=\frac{\varepsilon}{2}
\end{aligned}
$$

and the proof ends by noticing that $\varepsilon>0$ was arbitrarily fixed. 
Letting $A_{3}=\emptyset$ in Theorem 3 we get the assertion of Theorem 2. Actually, Theorem 2 can be proved via a direct argument based on Lemma 1.

Acknowledgement 1. The second author was partially supported by CNCSIS Grant 80/2005.

\section{REFERENCES}

[1] R. G. Bartle, A Modern Theory of Integration, Graduate Studies in Mathematics, Vol. 32, Amer. Math. Soc., Providence, R.I, 2001.

[2] N. Bourbaki, Fonctions d'une variable réelle (Théorie élémentaire), Hermann, Paris, 1949.

[3] A. M. Bruckner and J. L. Leonard, Derivatives, Amer. Math. Month., 73 (1966), no. 2, part $2,24-56$.

[4] A. Denjoy, Mémoire sur les nombres derivés des fonctions continues, Journal de Mathématiques Pures et Appliquées, 7th Series, Vol. 1 (1915), 105-240.

[5] J. Dieudonné, Foundations of Modern Analysis, Academic Press, New York and London, 1960.

[6] R. A. Gordon, The Integrals of Lebesgue, Denjoy, Perron, and Henstock, Graduate Studies in Mathematics, Vol. 4, Amer. Math. Soc., Providence, R.I., 1994.

[7] E. Hewitt and K. Stromberg, Real and Abstract Analysis, Springer-Verlag, New York, 1965.

[8] R. M. Moss and G. T. Roberts, A Creeping Lemma, Amer. Math. Month., 75 (1968), 649-651.

[9] C.P. Niculescu and F. Popovici, A Note on the Denjoy-Bourbaki Theorem, Real Anal. Exchange, 29 (2003/2004), 639-646.

[10] W. Rudin, Real and Complex Analysis, McGraw-Hill, New York, 3rd Edition, 1987.

[11] S. Saks, Theory of the Integral, Second ed., Monografje Matematyczne, Warszawa, 1937.

[12] P. L. Walker, On Lebesgue Integrable Derivatives, Amer. Math. Month., 84 (1977), 287-288.

Department of Mathematics, Rutgers University, NJ-08854, U.S.A.

E-mail address: ddutkay@math.rutgers.edu

Department of Mathematics, University of Craiova, Street A.I. Cuza 13, Craiova RO 200585, ROMANIA

E-mail address: cniculescu@central.ucv.ro

College Nicolae Titulescu, Braşov RO 500435, Romania

E-mail address: popovici.florin@yahoo.com 